\title{
ON THE BELIEVABILITY OF POLAR SPOTS
}

\author{
P. BRENDAN BYRNE \\ Armagh Observatory \\ College Hill, Armagh BT61 9DG, N. Ireland
}

\begin{abstract}
Polar spots in Doppler Imaging maps of rapidly rotating latetype stars, especially the active components of RS CVn binaries, represent a major break with the solar paradigm, within which spots are only found at low latitudes. I examine critically the evidence for polar spots and point out that they are particularly sensitive to a number of systematic effects relating to determining reference "zero-points".
\end{abstract}

\section{Introduction}

The technique of Doppler Imaging offers the potential to resolve in some detail spot distributions on active late-type stars. Since the seminal paper by Vogt and Penrod (1983) which imaged the spot distribution on the RS CVn star, V711 Tau (=HR1099), almost all such images have shown a dark polar cap. These have been attributed to large-scale spots including a symmetric distribution at the stellar pole.

Such spot distributions represent a fundamental break with the solar paradigm, in which spots are always found at latitudes between $\sim 10^{\circ}-40^{\circ}$. Of course, in view of the very great difference in magnetic activity between the Sun and the most active stars, there may be a fundamental difference in their field distributions. Indeed, Schüssler and Solanki (1992) and Schüssler (these proceedings) have argued that, in the presence of differential rotation in the most rapidly rotating, and therefore magnetically active, stars, magnetic field, originating at the base of the convection zone at low latitude, will appear at the stellar surface at higher latitudes due to the influence of Coriolis forces. This mechanism will not generate polar spots, however.

In this review we examine critically the case for the reality of these polar spots and point out some possible problems with their acceptance. 

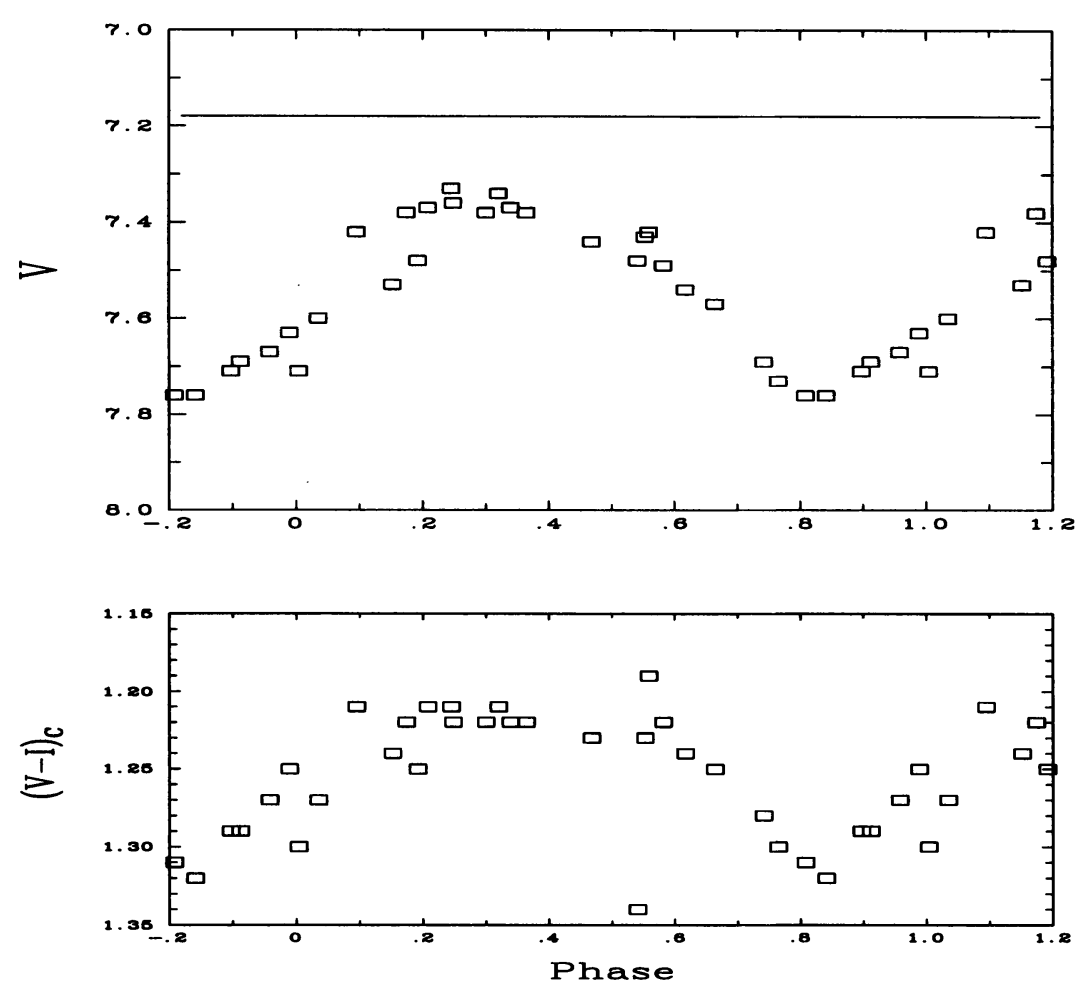

Figure 1. The 1989 season light and colour curves for the RS CVn star, II Peg (Byrne, in prep). The solid horizontal line represents the record brightest observation of Chugainov (1976).

\section{Photometry}

Although the evidence for polar spots comes primarily from Doppler Imaging, there are aspects of the phenomenon to which photometry can contribute information.

Large amplitude light curves (e.g. Byrne \& Marang, 1987, Strassmeier $\&$ Olah, 1992) give clear evidence that large concentrations of low latitude dark spots must exist on many active late-type stars (Fig. 1). The amplitudes of the largest of these are such that the causal spots must cross the line-of-sight to the observer, i.e. the least limb darkened portion of the disk, in order to give rise to the observed light curve amplitude (Byrne \& Marang, 1987). In systems such as II Peg, for instance, in which the inclination of the rotational axis is close to $90^{\circ}$, such spot distributions cannot be at high latitude.

In Fig. 2 we illustrate this point by plotting the synthetic $\mathrm{V}$ light curves for a circular spot of radius $30^{\circ}\left(T_{\text {star }}=4650 \mathrm{~K}, T_{\text {spot }}=3000 \mathrm{~K}, i=90^{\circ}\right)$ at 


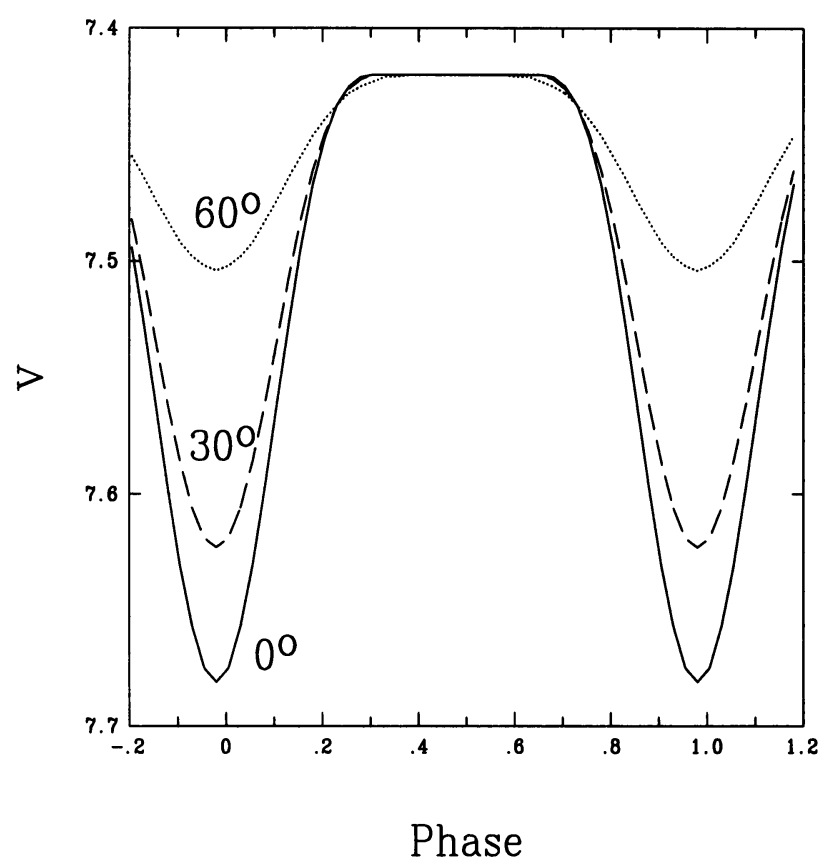

Figure 2. An illustration of the effect of spot latitude on the darkening produced by a circular spot of fixed radius $\left(30^{\circ}\right)$ for three different latitudes on a star with $i=90^{\circ}$.

three different latitudes, viz. $0^{\circ}, 30^{\circ}$, and $60^{\circ}$. The darkening effect of the spot decreases enormously at higher latitudes due to the combined effects of geometric projection of the spot and limb darkening of the underlying disk photosphere masked by the spot. Thus to account for the largest amplitude light curves $(\Delta V \geq 0.3)$ low latitude spots are difficult to avoid.

There is also, however, evidence from photometry, for a non-modulating spot distribution (i.e. one visible at all phases) whose total area is a very significant fraction of the stellar disk. Such evidence arises from a comparison of historical maxima with current data. For instance, in the case of the light curve in Fig. 1, the maximum light observed is $V=7.3$. However, a previous data set recorded a maximum of $\mathrm{V}=7.18$ in 1974 Chugainov (1976). So, unless the luminosity of II Peg changed, it seems that there is a spot distribution present which is visible at all phases and so does not modulate the light. One possible location for this is at the stellar pole (see e.g. Rodonó et al. 1986). However, photometry alone cannot distinguish between this possibility and a quasi-uniform low latitude band of spots. 
Recent results using a pair of $\mathrm{TiO}$ bands as both a temperature and area indicator (Neff et al. 1995, O'Neal et al., this proceedings) have suggested that the true unspotted magnitude for the star II Peg is even brighter than that observed by Chugainov. Their results suggest an unspotted brightness of $\mathrm{V}=6.8$.

\section{Doppler Imaging}

As explained elsewhere in these proceedings (see e.g. Vogt) Doppler Imaging relies on the deformation of a rotationally broadened photospheric line profile resulting from the non-uniform distribution of continuum brightness across the stellar disk caused by the presence of spots. In the case of nonpolar spots the speed of passage of such features across the mapping profile indicates their latitude, while their intensity amplitudes give information on their total area. Polar spots, however, are stationary on the mapping profile and so their detection relies on an accurate knowledge of the line profile of the unspotted photosphere.

Reference non-spotted line profiles are usually obtained in one of two ways. On the one hand use is made of the profile of a slowly rotating, assumed unspotted, star of the same spectral type, artificially spun up to the rotation of the spotted star. On the other a line profile is calculated from a plane parallel, LTE model atmosphere. In either case it should be borne in mind that the atmospheres of rapidly rotating active stars are very different from those of slowly rotating inactive stars.

The most important points of difference are the following.

- Active stars' atmospheres are highly magnetic

- They undergo large amounts of non-radiative heating which may include their upper photospheres

- Their photospheres are bathed in a high temperature back-heating flux from the corona

- The most rapid rotators suffer a reduced effective gravity at their equators relative to their poles

- Many, the shorter period RSCVn systems in particular, suffer tidal effects.

All of the above have the ability to distort template profiles as calculated from LTE, plane-parallel model atmospheres or deduced from spun-up quiescent atmospheres.

The high filling factors and strong fields implied by recent studies (Saar, this volume) introduce a preferred direction in active atmospheres. This in turn brings with it the possibility of non-isotropic micro- and macroturbulent velocity fields, an effect recently evaluated in an ad-hoc manner by Unruh and Cameron (1995). Unruh and Cameron found that polar spots 
could be generated in test reconstructions of non-polar spot stars by neglect of such effects.

High density, non-radiatively heated chromospheres in active stars are the result of a steep temperature rise above the photosphere. Such atmospheric structures can lead to non-LTE effects, especially in the cores of strong lines of neutral species, normally considered as pure photospheric lines (Houdebine, this proceedings). Core filling can deform the reference line profile used in Doppler imaging to mimic stationary, polar features.

Unruh and Cameron also drew attention to the potential of non-standard limb darkening laws for generating non-existent polar features. In this regard we note that effects of equatorial levitation due to rapid rotation, tidal effects and magnetic evacuation can all modify standard limb darkening laws.

\section{Conclusions}

Doppler Imaging offers an extremely powerful technique to image the surfaces of active late-type stars. Nevertheless it may be subject to "zeropoint" errors through neglect of various effects in preparing template reference spectra of the underlying non-spotted photosphere. Symmetric polar features are particularly prone to such effects. It is important, therefore, to evaluate thoroughly the effects of various line deformations on the resulting images. In the light of the points raised here the recent work of Unruh and Cameron is especially welcome.

Acknowledgements: Research at Armagh Observatory is supported by a Grant-in-Aid from the Department of Education for Northern Ireland.

\section{References}

Byrne, P.B., Marang, F. 1987, Irish AJ, 18, 84

Chugainov, P.F. 1976, Izv. Krymskoi Obs. 54, 89

Neff, J.E., O'Neal, D., Saar, S.M. 1995, ApJ, 452, 879

Rodonó, M., Cutispoto, G., Pazzani, V., Catalano, S., Byrne, P.B., Doyle, J.G., Butler, C.J., Andrews, A.D., Blanco, C., Marilli, E., Linsky, J.L., Scaltriti, F., Busso, M., Cellino, A., Hopkins, J.L., Okazaki, A., Hayashi, S.S., Zeilik, M., Helston, R., Henson, G., Smith, P., Simon, T. 1986, A\&A, 165, 135

Schüssler, M, Solanki, S.K. 1992, A\&A, 264, 13

Strassmeier, K.G., Olah, K. 1992, A\&A, 259, 595

Unruh, Y., Cameron, A.C., 1995, MNRAS, 273, 1

Vogt, S.S., Penrod, G.D., 1983, PASP, 95, 565 
Showdown! "To be polar or not ..." that's the question. Klaus Strassmeier, pro (left) and Brendan Byrne, con (right).
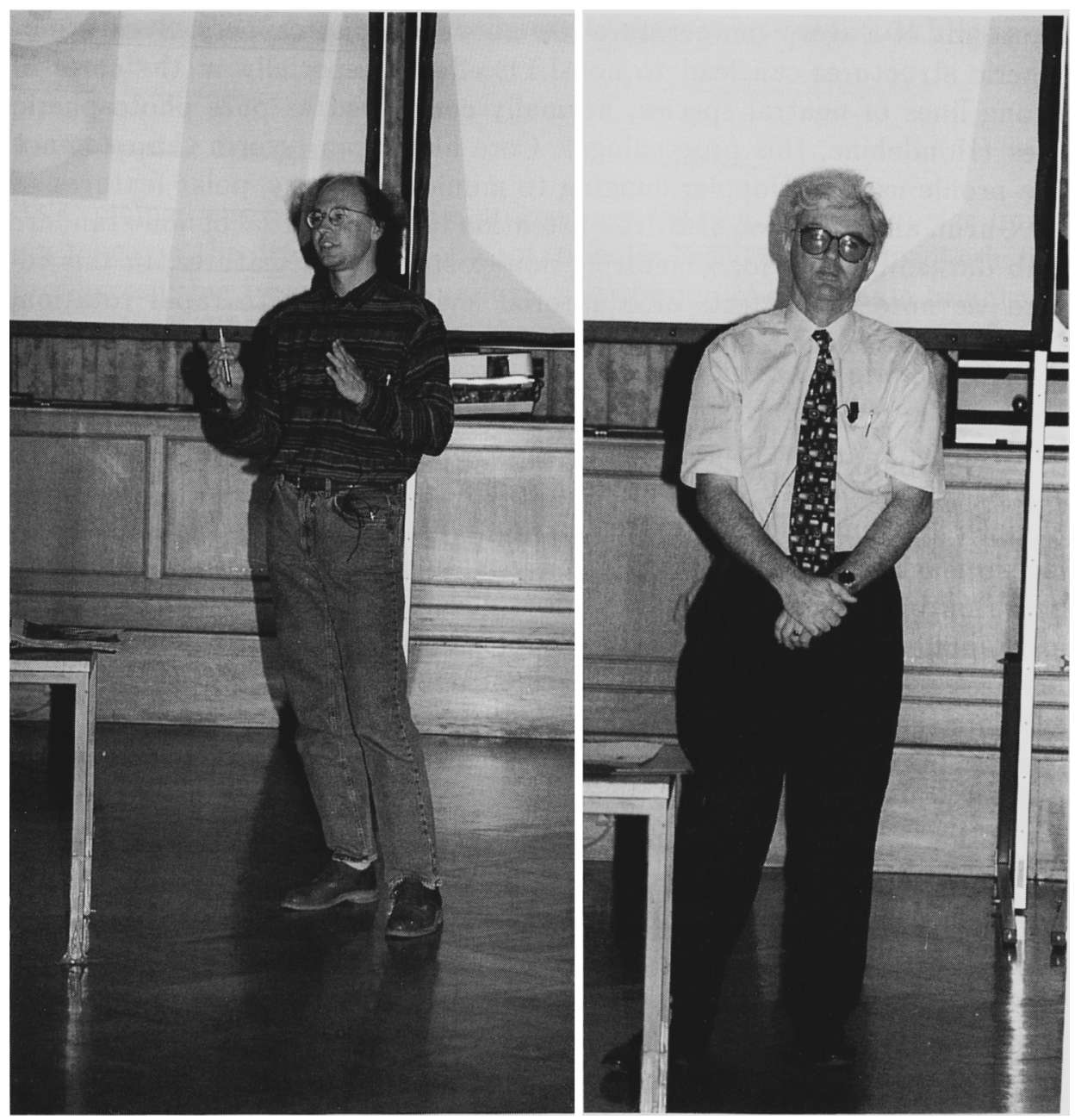